Publ. RIMS, Kyoto Univ.

33 (1997), 751-764

\title{
An Upper Bound for the Characteristic Variety of an Induced $\mathscr{D}$-Module
}

By

\author{
Carlos MEnEZES*
}

\begin{abstract}
We generalise the $\operatorname{Car}_{Y}^{z}(\mathfrak{M})$ upper bound of Laurent \& Schapira [LS87] for the characteristic variety of the induced system of a coherent $\mathscr{D}_{X}$-module $\mathfrak{M}$ on a hypersurface $Y$ of $X$. to the case where $Y$ is a smooth submanifold of $X$ of arbitrary codimension
\end{abstract}

\section{Contents}

1. Introduction

2. Definition of $\operatorname{Car}\left(\mathfrak{M}_{Y}^{\bullet}\right)$

3. Differential operators on a holomorphic vector bundle

4. Definition of $\operatorname{Car}_{Y}^{\mathbf{Z}}(\mathfrak{M})$

5. The case where $\mathfrak{M}$ is the module defined by one operator

6. The case where $\mathfrak{M}$ is the module defined by a coherent ideal

7. Main Theorem

References

\section{Introduction}

Given a complex analytic manifold $X$ and a smooth submanifold $Y$ of $X$, let $T^{*} X \rightarrow X$ be the cotangent bundle of $X, T^{*} Y \rightarrow Y$ the cotangent bundle of $Y . T_{Y} X$ $\stackrel{\lambda}{\rightarrow} Y$ the normal bundle of $Y$ in $X, T_{Y}^{*} X \stackrel{\lambda^{*}}{\rightarrow} Y$ the conormal bundle of $Y$ in $X$, and let $\rho$ and $\bar{\omega}$ be the maps cannonically associated to the immersion $Y \stackrel{\jmath}{\rightarrow} X$ :

\footnotetext{
Communicated by M. Kashiwara, November 21. 1995. Revised March 4, 1997.

1991 Mathematics Subject Classification: 58G7; Secondary 58G20.

Key words and phrases: $\mathscr{D}$-modules, Characteristic Variety.

Partially supported by Centro de Matematica da Universidade do Porto and Projecto Analise Microlocal e Teoria das Singularidades PBIC/C/MAT/2153/95.

*Dept. Mat. Pura, Fac. Ciencias Univ. Porto, Praça Gomes Teixeira, 4050 Porto, Portugal, and. Centro de Matemática da Univ. Portugal.

E-mail address: cmmenez@fc1.fc.up.pt
} 


$$
T^{*} Y \stackrel{\rho}{\leftarrow} Y \times_{X} T^{*} X \stackrel{\bar{\omega}}{\rightarrow} T^{*} X .
$$

Let $\mathscr{O}_{X}$ be the structural sheaf of $X, \mathscr{F}_{Y}$ the defining ideal of $Y, \mathscr{O}_{Y}=\mathscr{O}_{X} / \mathscr{E}_{Y}$ the structural sheaf of $Y, \mathscr{D}_{X}$ the sheaf of holomorphic differential operators of finite order in $X, \mathscr{D}_{X \mid Y}$ the restriction of $\mathscr{D}_{X}$ to $Y$, and let

$$
\mathscr{D}_{Y \rightarrow X}=\mathscr{O}_{Y} \bigotimes_{\mathscr{O}_{X}} \mathscr{D}_{X \mid Y}=\mathscr{D}_{X} / \mathscr{J}_{Y} \mathscr{D}_{X}
$$

be the transfer bimodule from $Y$ to $X$. Given a coherent $\mathscr{D}_{X}$-Module $\mathfrak{M}$. let $\mathfrak{M}_{Y}^{\circ}=\mathscr{D}_{Y \rightarrow X} \stackrel{\mathbb{L}}{\otimes}_{\mathscr{D}_{\lambda}} \mathfrak{M}$ be the induced $\mathscr{D}_{Y}$-Module in $Y$. Define

$$
\mathfrak{M}_{Y}^{k}=\mathbb{H}^{-k}\left(\mathfrak{M}_{Y}^{\circ}\right)=\mathbb{T} \mathbb{D} \mathbb{P}_{k}^{\mathscr{D}_{X}}\left(\mathscr{D}_{Y \rightarrow X}, \mathfrak{M}\right) .
$$

Kashiwara [Ka83a] proved that, if $\mathfrak{M}$ is non-characteristic for $Y$, then

- the cohomology of the complex $\mathfrak{M}_{Y}^{\circ}$ is concentrated in degree 0 ;

- $\mathfrak{M}_{Y}^{0}$ is a coherent $\mathscr{D}_{Y}$-module;

- $\operatorname{Car}\left(\mathfrak{M}_{Y}^{0}\right)=\rho \bar{\omega}^{-1} \operatorname{Car}(\mathfrak{M})$.

Consider now in $\mathscr{D}_{X \mid Y}$ the Kashiwara $[\mathrm{Ka} 83 \mathrm{~b}] V$-filtration associated to the embedding $Y \stackrel{j}{\rightarrow} X$ and defined in degree $k$ by

$$
F_{Y}^{k} \mathscr{D}_{X}=\left\{P \in \mathscr{D}_{X \mid Y}: P \mathscr{g}_{Y}{ }^{l} \subset \mathscr{g}_{Y}{ }^{l-k} \quad \forall l \in \mathbb{N}\right\},
$$

and let $F_{Y}^{k} \mathscr{D}_{Y \rightarrow X}=\frac{F^{k} \mathscr{T}_{X \mid Y}}{\bar{f}_{Y} \cap \mathscr{D}_{X \mid 1}}$ be the degree $k$ of the corresponding $F_{Y} \mathscr{D}_{Y \rightarrow X}$ quotient filtration.

Let $\mathfrak{M}$ be an arbitrary coherent $\mathscr{D}_{X}$-module not necessarily non characteristic for $Y$. In [LS87] Laurent \& Schapira proved that

- $\mathfrak{M}_{Y}^{k}$ is a union of an increasing sequence of coherent $\mathscr{D}_{Y}$-modules.

So they could define the notion of characteristic variety of $\mathfrak{M}_{Y}^{\circ}, \operatorname{Car}\left(\mathfrak{M}_{Y}^{\circ}\right)$. Moreover by [Sch85] the sheaf of graded rings $g r_{Y}\left(\mathscr{D}_{X}\right)$ is isomorphic to the subsheaf $\lambda_{*} \mathscr{D}_{\left[T_{Y} X\right]}$ of rings of holomorphic differential operators of finite order on $T_{Y} X$ that are algebraic in the fibers, and if $F_{Y} \mathfrak{M}$ is a $F_{Y} \mathscr{D}_{X}$-good filtration on $\mathfrak{M}$ then the graded module of $\mathfrak{M}$ for this filtration, $g r_{Y}(\mathfrak{M})$, is a $g \gamma_{Y}\left(\mathscr{D}_{X}\right)$-coherent module. Denoting by $\widehat{C}_{T_{Y} X}(\mathfrak{M}) \subset T^{*} T_{Y} X$ the formal microcharacteristic variety of $\mathfrak{M}$ along $Y$, i.e. the characteristic variety of $\mathscr{D}_{T_{Y} X} \otimes_{\lambda^{-1} g r_{1}\left(\mathscr{D}_{\lambda}\right)} g r_{Y}(\mathfrak{M})$, it was proved in [LS87] that

$$
\text { - } \operatorname{Car}\left(\mathfrak{M}_{Y}^{\circ}\right) \subset T^{*} Y \cap \widehat{C}_{T_{Y} X}(\mathfrak{M}) \text {. }
$$

Moreover, when $Y$ is smooth embedded hypersurface of $X$, in [LS87] was defined a new subset of $T^{*} Y$, denoted $\operatorname{Car}_{Y}^{Z}(\mathfrak{M})$, and it was proved that

$$
\text { - } \operatorname{Car}\left(\mathfrak{M}_{Y}^{\circ}\right) \subset \operatorname{Car} Z \mathbf{Z}(\mathfrak{M}) \subset T^{*} Y \cap \widehat{C}_{T_{Y} X}(\mathfrak{M}) \text {. }
$$

providing a better upper bound for $\operatorname{Car}\left(\mathfrak{M}_{Y}^{\circ}\right)$.

The aim of this work is to generalize the construction of the $\operatorname{Car}_{Y}^{Z}(\mathfrak{M})$ of [LS87] to the case where $Y$ is a smooth embedded submanifold of $X$ of arbitrary codimension.

To finish this introductory section some of the above globally defined objects are computed in a special coordinate system. 
The above objects in local coordinates. Let $(y, t)=\left(y_{1}, \ldots, y_{m-q}, t_{1}, \ldots\right.$, $\left.t_{q}\right)$ be a local coordinate system in $X$ such that $Y=\{(y, t): t=0\}$. Then:

$$
T_{Y} X=\left\{(y, \tau): y \in \mathbb{C}^{m-q}, \tau \in \mathbb{C}^{q}\right\},
$$

and

$$
\mathscr{D}_{Y \rightarrow X} \simeq \frac{\mathscr{D}_{X}}{t_{1} \mathscr{D}_{X}+\cdots+t_{q} \mathscr{D}_{X}} .
$$

Let $\delta^{\alpha}=\delta^{\left(\alpha_{1}, \ldots, \alpha q\right)}$ be the image of $\partial_{t_{1}, \ldots,}^{\alpha_{1}} \partial_{t q}^{\alpha_{q}} \in \mathscr{D}_{X}$ by the canonical projection $\mathscr{D}_{X} \rightarrow \mathscr{D}_{Y \rightarrow X}=\frac{\mathscr{D}_{X}}{l_{1} \mathscr{D}_{1}+\cdots+l_{q} \mathscr{D}_{Y}}$. Then

$$
F_{Y}^{k} \mathscr{D}_{Y \rightarrow X} \simeq \underset{|\alpha| \leq k}{\bigoplus} \mathscr{D}_{Y} \delta^{\alpha}
$$

and

$$
\mathscr{D}_{Y \rightarrow X} \simeq \underset{k \geq 0|\alpha|=k}{\bigoplus} \mathscr{D}_{Y} \delta^{\alpha}
$$

Acknowlegments. I want to thank Professor T. Monteiro Fernandes for her continuing support and encouragement during the preparation of this paper. I also want to thank Professor M. Kashiwara for some discussions about the results of this paper.

\section{Definition of $\operatorname{Car}\left(\mathfrak{M}_{Y}^{\bullet}\right)$}

Proposition 2.1. [LS87]. Let $\left(X, \mathscr{O}_{X}\right)$ be a complex analytic manifuld and let $Y$ be a smooth submanifold of $X$. Let $\mathfrak{M}$ be a coherent $\mathscr{D}_{X^{-}}$module. Then the $\mathscr{D}_{Y^{-}}$modules $\mathfrak{M}_{Y}^{k}$ may be locally written as a union of an increasing sequence of coherent $\mathscr{D}_{Y^{-} \text {modules. }}$

Proof. Consider a local finite type free resolution of $\mathfrak{M}$ :

$$
0 \rightarrow \mathscr{D}_{X} m^{p} \stackrel{A p-1}{\longrightarrow} \cdots \stackrel{A_{0}}{\longrightarrow} \mathscr{D}_{X} m^{0} \longrightarrow \mathfrak{M} \rightarrow 0 .
$$

where $A_{\imath}(i=0, \ldots, p-1)$ is a $m_{\imath+1} \times m_{\imath}$ matrix of differential operators that acts on the right of $\mathscr{D}_{X}{ }^{m^{t+1}}$. Tensoring (1) on the left by $\mathscr{D}_{Y \rightarrow X} \otimes_{\mathscr{D}_{X}}$ we get the complex

$$
\left(\mathscr{D}_{Y \rightarrow X}\right)^{m^{p}} \rightarrow \cdots \rightarrow\left(\mathscr{D}_{Y \rightarrow X}\right)^{m^{0}}
$$

which is quasi-isomorphic to $\mathfrak{M}_{Y}^{\odot}$. Then

$$
\begin{aligned}
\operatorname{Ker}\left(A_{\imath-1}\right) & =\bigcup_{k \in \mathbf{N}} \operatorname{Ker}\left(F_{Y}^{k} \mathscr{D}_{Y \rightarrow X}{ }^{m^{t} \rightarrow \mathscr{D}_{Y \rightarrow X}}{ }^{m^{t-1}}\right) \\
& =\bigcup_{k \in \mathbf{N}} \operatorname{Ker}\left(F_{Y}^{k} \mathscr{D}_{Y \rightarrow X}{ }^{\left.m^{t} \rightarrow F_{Y}^{k+l} \mathscr{D}_{Y \rightarrow X} m^{m^{t-1}}\right)}\right.
\end{aligned}
$$

for a big enough $l \geq 0$. Setting 


$$
K_{\imath}(k)=\mathbb{K} \operatorname{err}\left(F_{Y}^{k} \mathscr{D}_{Y \rightarrow X} m^{i} \rightarrow F_{Y}^{k+l} \mathscr{D}_{Y \rightarrow X} m^{t-1}\right)
$$

we have that $K_{\imath}(k) \subset K_{\imath}(k+1)$ and that $K_{\imath}(k)$ is a coherent $\mathscr{D}_{Y}$-module. This proves that $\operatorname{Ker}\left(A_{i-1}\right)$ is a union of an increasing sequence of coherent $\mathscr{D}_{Y}$-modules. On the other hand we have:

$$
\begin{aligned}
& \operatorname{Imm}\left(A_{\imath}\right)=\bigcup_{k \in \mathbb{N}} \operatorname{Im}\left(\mathscr{D}_{Y \rightarrow X}{ }^{m^{i+1}} \rightarrow \mathscr{D}_{Y \rightarrow X} m^{m^{t}}\right) \cap F_{Y}^{k} \mathscr{D}_{Y \rightarrow X} m^{m^{t}} \\
& =\underset{k \in \mathbb{N} u \in \mathbb{N}}{\cup} \operatorname{Im}\left(F_{Y}^{l} \mathscr{D}_{Y \rightarrow X} m^{m^{t+1}} \rightarrow \mathscr{D}_{Y \rightarrow X}{ }^{m^{2}}\right) \cap F_{Y}^{k} \mathscr{D}_{Y \rightarrow X}{ }^{m^{t}} .
\end{aligned}
$$

Setting

$$
I_{l}(k)=\bigcup_{l \in \mathbb{N}} \mathbb{I} \operatorname{Im}\left(F_{Y}^{l} \mathscr{D}_{Y \rightarrow X}{ }^{m^{t-1}} \rightarrow \mathscr{D}_{Y \rightarrow X} m^{i}\right) \cap F_{Y}^{k} \mathscr{D}_{Y \rightarrow X}{ }^{m^{l}},
$$

we see that $I_{i}(k)$ is a union of an increasing sequence of coherent sub- $\mathscr{D}_{Y^{-}}$ modules of the coherent $\mathscr{D}_{Y}$-module $F_{Y}^{k} \mathscr{D}_{Y \rightarrow X}{ }^{m^{\prime}}$. Being $\mathscr{D}_{Y}$ a noetherian sheaf of rings, $I_{t}(k)$ is a coherent $\mathscr{D}_{Y}$-module. Finally we have $\operatorname{Im}\left(A_{t}\right)=\cup_{k \in \mathbb{N}} I_{t}(k)$ and $I_{l}(k) \subset I_{i}(k+1)$. Hence it follows that $\operatorname{Im}\left(A_{\imath}\right)$ is also a union of an increasing sequence of coherent $\mathscr{D}_{Y}$-modules.

Now let $\mathfrak{R}$ be a left $\mathscr{D}_{Y}$-module, locally a union of an increasing sequence of coherent $\mathscr{D}_{Y}$-modules $\left(\mathfrak{R}_{k}\right)_{k \in \mathbb{N}}$. Then the subset

$$
\operatorname{Car}(\mathfrak{R}):=\cup_{k \in \mathbb{N}} \operatorname{Car}\left(\mathfrak{\Re}_{k}\right)
$$

does not depend on the sequence $\left(\mathfrak{N}_{k}\right)_{k \in \mathbf{N}}$ and is called the Characteristic Variety of $\mathfrak{R}$.

If $0 \longrightarrow \mathfrak{N}^{\prime} \longrightarrow \mathfrak{R} \longrightarrow \mathfrak{N}^{\prime \prime} \longrightarrow 0$ is an exact sequence of $\mathscr{D}_{Y^{-}}$modules of the preceding type then

$$
\operatorname{Car}(\mathfrak{N})=\operatorname{Car}\left(\mathfrak{\mathfrak { Z } ^ { \prime }}\right) \cup \operatorname{Car}\left(\mathfrak{R}^{\prime \prime}\right)
$$

Defimition 2.2. If $\mathfrak{N}^{\circ}$ is a bounded complex of $\mathscr{D}_{Y^{-}}$modules such that the cohomology groups are $\mathscr{D}_{Y}$-modules of the preceding type the characteristic variety of the complex $\mathfrak{R}^{\circ}$ is defined to be the following subset of $T^{*} Y$ :

$$
\operatorname{Car}\left(\mathfrak{N}^{\circ}\right)=\underset{j \in \mathbb{Z}}{\cup} \operatorname{Car}\left(\mathbb{H}^{\prime}\left(\mathfrak{N}^{\circ}\right)\right) .
$$

In particular if $\mathfrak{M}$ is a coherent $\mathscr{D}_{X}$-module, then the characteristic variety of $\mathfrak{M}_{Y}^{\circ}$ is the following subset of $T^{*} Y$

$$
\operatorname{Car}\left(\mathfrak{M}_{Y}^{\circ}\right):=\cup_{j \in \mathbb{N}} \operatorname{Car}\left(\mathbb{H}^{-j}\left(\mathfrak{M}_{Y}^{\circ}\right)\right)
$$

\section{Differential Operators on a Holomorpic Vector Bundle}

Given a holomorphic vector bundle of rank $q$ over the complex analytic manifold $Y$, 


$$
\Lambda \stackrel{\lambda}{\longrightarrow} Y
$$

let $\theta=e_{\lambda}$ be the Euler-vector field of $\Lambda$. Given an integer $k$ let

$$
\mathscr{O}_{[\Lambda]}[k]=\left\{f \in \mathscr{O}_{\Lambda}: \theta f=k f\right\}
$$

be the sheaf of holomorphic functions on $\Lambda$ that are homogeneous of degree $k$ in the fibers, and let

$$
\mathscr{D}_{[A]}[k]=\left\{P \in \mathscr{D}_{\Lambda}:[\theta, P]=-k P\right\}
$$

be the sheaf of holomorphic differential operators on $\Lambda$ that are homogeneous of degree $k$ in the fibers. The following proposition is clear:

Proposition 3.1. [LS87] The map $\lambda_{*} \mathscr{O}_{[\Lambda]}[0] \stackrel{\rho}{\longrightarrow} \mathscr{O}_{Y}, f \mapsto f_{\mid Y}$ is an isomorphism of $\mathscr{O}_{Y^{-}}$modules.

The sheaf $\lambda_{*} \mathscr{D}_{[A]}[0]$ acts on the left of $\lambda_{*} \mathscr{O}_{[\Lambda]}[0]$ and so also on $\mathscr{O}_{Y \text {. This }}$ defines a morphism of sheaf of rings $\lambda_{*} \mathscr{D}_{[\Lambda]}[0] \stackrel{\rho}{\rightarrow} \mathscr{D}_{Y}$.

If $(y, t)$ is a local trivialization of $\Lambda$ such that $\lambda(y, t)=y$, then the differential operators $P \in \mathscr{D}_{[\Lambda]}[k]$ are those that may be written in that coordinate system in the form:

$$
P=\sum_{|\alpha|-|\beta|=k} P_{\alpha, \beta}\left(y, \partial_{y}\right) t^{\alpha} \partial_{t}^{\beta} .
$$

In particular the differential operators $P \in \mathscr{D}_{\Lambda}[0]$ are those that may be written in the form:

$$
P=\sum_{|\alpha|=|\beta|} P_{\alpha, \beta}\left(y, \partial_{y}\right) t^{\alpha} \partial_{t}^{\beta}
$$

and we have

$$
\rho(P)=P_{0,0}\left(y, \partial_{y}\right) .
$$

Thus, locally, $\lambda_{*} \mathscr{D}_{[\Lambda]}[0]$ is identified to

$$
\mathscr{D}_{Y}\langle\theta\rangle:=\mathscr{D}_{Y}\left[\theta_{11}, \theta_{12} \ldots, \theta_{1 q}, \ldots, \theta_{q 1}, \ldots, \theta_{q q}\right] /\{\text { commutation relations }\}
$$

where, by definition, $\theta_{i},=t_{t} \partial_{t}$, and the commutation relations between the variables $\theta_{l j}$ are the following ones:

$$
\left[\theta_{i j}, \theta_{k l}\right]=\left[t_{\imath} \partial_{t}, t_{k} \partial_{t l}\right]= \begin{cases}0 & \text { if } j \neq k \text { and } i \neq l \\ \theta_{\imath \jmath} & \text { if } j=k \text { and } i \neq l \\ \theta_{t \imath}-\theta_{\jmath \jmath} & \text { if } j=k \text { and } i=l \\ -\theta_{k s} & \text { if } j \neq k \text { and } i=l\end{cases}
$$

In particular, locally, $\rho$ is identified to $\rho\left(P\left(y, \partial_{y}, \theta_{\imath}\right)\right)=P\left(y, \partial_{y}, 0\right)$

If $\mathfrak{N}$ is a coherent $\lambda_{*} \mathscr{D}_{\Lambda]}[0]$-module the coherent $\mathscr{D}_{Y}$-module $\rho(\mathfrak{N})$ is 
defined by "extension" of scalars:

$$
\rho(\mathfrak{N})=\mathscr{D}_{Y} \otimes_{\lambda_{*} \mathscr{D}_{[M]}[0]} \mathfrak{N}
$$

thus having a characteristic variety $\operatorname{Car}(\rho(\mathfrak{N}))$ in its own right, which is an involutive analytic subset of $T^{*} Y$.

\section{Proposition 3.2.}

(a) If $0 \longrightarrow \mathfrak{N} \longrightarrow \mathfrak{N} \longrightarrow \mathfrak{N}^{\prime \prime} \longrightarrow 0$ is an exact sequence of coherent $\lambda * \mathscr{D}_{[1]}[0]$-modules then

$$
\operatorname{Car}(\rho(\mathfrak{N}))=\operatorname{Car}\left(\rho\left(\mathfrak{N}^{\prime}\right)\right) \cup \operatorname{Car}\left(\rho\left(\mathfrak{N}^{\prime \prime}\right)\right) .
$$

(b) If $\mathscr{g}$ is coherent ideal of $\lambda_{*} \mathscr{D}_{[A]}[0]$ and if $\mathfrak{\Re}=\lambda_{*} \mathscr{D}_{[A]}[0] / \mathscr{\&}$ then

$$
\operatorname{Car}(\rho(\mathfrak{N}))=\left\{y^{*} \in T^{*} Y: \forall P \in \mathscr{J} \sigma(\rho(P))\left(y^{*}\right)=0\right\}
$$

Proof. The problem being of local character we can set

$$
\lambda_{*} \mathscr{D}_{[\Lambda]}[0]=\mathscr{D}_{Y}\langle\theta\rangle \text {. }
$$

Let $I$ be the left ideal of $\mathscr{D}_{Y}\langle\theta\rangle$ generated by $\theta_{11}, \theta_{12}, \ldots, \theta_{1 q}, \ldots, \theta_{q 1}, \ldots, \theta_{q q}$. Then:

$$
\begin{aligned}
& \circ \mathscr{D}_{Y} \simeq \frac{\mathscr{D}_{Y}\langle\theta\rangle}{I} \\
& \circ \theta_{i j} \in I \quad \forall i, j,
\end{aligned}
$$

and the commutation relations (2) give

$\circ \theta_{\imath} \in I^{k}$ if $i \neq j$

$\circ \theta_{\imath \imath}-\theta_{j,} \in I^{k} \forall i, j$

$\circ \theta_{i l}^{k}-\theta_{j j}^{k} \in I^{k} \forall i, j \forall k \in \mathbb{N}$.

Let $F \mathscr{D}_{Y}\langle\theta\rangle$ be the non-separated filtration on $\mathscr{D}_{Y}\langle\theta\rangle$ defined by

$$
F_{k} \mathscr{D}_{Y}\langle\theta\rangle= \begin{cases}\mathscr{D}_{Y}\langle\theta\rangle & \text { if } k \geq 0 \\ I^{-k} & \text { if } k<0\end{cases}
$$

The properties of $\mathscr{D}_{Y}\langle\theta\rangle$ listed above imply that the graded ring of $\mathscr{D}_{Y}\langle\theta\rangle$ for this filtration is isomorphic to the ring of polynomials $\mathscr{D}_{Y}[\bar{\theta}]$ in one variable $\bar{\theta}$ and with coefficients in $\mathscr{D}_{Y}$, where $\bar{\theta}$ is the image of all the $\theta_{\imath \imath} \in I^{1}(i=1, \ldots, q)$ in the quotient $I^{1} / I^{2}$.

As $g r \mathscr{D}_{Y}\langle\theta\rangle \simeq \mathscr{D}_{Y}[\bar{\theta}]$ is a noetherian graded ring and

$$
F_{0} \mathscr{D}_{Y}\langle\theta\rangle=\mathscr{D}_{Y}\langle\theta\rangle
$$

is a noetherian filtered ring, proposition 1.1 .8 of Chap. II of [Sch85] implies that the filtration $F \mathscr{D}_{Y}\langle\theta\rangle$ is a noetherian one.

Now let $g r \mathscr{D}_{Y}\langle\theta\rangle$ be filtered by the order of holomorphic differential operators in $Y$.

If $\mathfrak{N}$ is a coherent $\mathscr{D}_{Y}\langle\theta\rangle$-module equipped with a good $F \mathscr{D}_{Y}\langle\theta\rangle$-filtration the graded module of $\mathfrak{N}$ for this filtration, $g r(\mathfrak{N})$, is a graded coherent 
$\mathscr{D}_{Y}[\bar{\theta}]$-module whose characteristic variety $\operatorname{Car}(\mathrm{gr} \mathfrak{\Re})$ is an analytic subset of $T^{*}(Y) \times \mathbf{C}$.

By Proposition 1.3.1 of Chap. II of [Sch85], the characteristic variety Car $(g r \Re)$ is independent of the choice of the good filtration on $\mathfrak{N}$ and the map that sends $\mathfrak{N}$ to $\operatorname{Car}(\mathrm{gr} \mathfrak{N})$ is an additive map, that is, if $0 \longrightarrow \mathfrak{N}^{\prime} \longrightarrow \mathfrak{N} \longrightarrow \mathfrak{N}^{\prime \prime} \longrightarrow 0$ is an exact sequence of coherent $\mathscr{D}_{Y}\langle\theta\rangle$-modules then $\operatorname{Car}(\operatorname{gr} \mathfrak{N})=\operatorname{Car}\left(\operatorname{gr} \mathfrak{N}^{\prime}\right) \cup$ $\operatorname{Car}\left(g r \mathfrak{N}^{\prime \prime}\right)$.

Hence, to prove the first part of the proposition it is enough to prove that $\operatorname{Car}(\operatorname{gr} \mathfrak{M})=\operatorname{Car}\left(\frac{\mathfrak{R}}{\mathfrak{N}}\right) \times \mathbb{C}$.

Suppose that $\mathfrak{N}$ is a coherent $\mathscr{D}_{Y}\langle\theta\rangle$-module. Then the filtration on $\mathfrak{R}$ defined by

$$
\mathfrak{N}_{k}= \begin{cases}\mathfrak{N} & \text { if } k \geq 0 \\ I^{-k} \mathfrak{N} & \text { if } k<0\end{cases}
$$

is a good filtration, and the graded module of $\mathfrak{N}$ for this filtration is

$$
g r(\mathfrak{N})=\bigoplus_{k \geq 0}=\frac{I^{k} \mathfrak{N}}{I^{k+1} \mathfrak{N}}
$$

So, for all $k \in \mathbb{Z}, \frac{I^{k} \mathfrak{R}}{I^{k+1} \mathscr{R}}$ is a coherent $\mathscr{D}_{Y}$-module and we have a surjective morphism of coherent $\mathscr{D}_{Y}$-modules

$$
\frac{\mathfrak{N}}{I_{\mathfrak{N}}} \stackrel{\bar{\theta}^{k}}{\longrightarrow} \frac{I^{k \mathfrak{N}}}{I^{k+1} \mathfrak{N}}
$$

Thus

$$
\operatorname{Car}\left(\frac{I^{k} \mathfrak{N}}{I^{k+1} \mathfrak{N}}\right) \subset \operatorname{Car}\left(\frac{\mathfrak{N}}{I \mathfrak{N}}\right) \subset T^{*} Y
$$

and

$$
\operatorname{Car}(g r \mathfrak{N})=\left(\bigoplus_{k \geq 0} \operatorname{Car}\left(\frac{I^{k} \mathfrak{N}}{I^{k+1} \mathfrak{\Re}}\right)\right) \times \mathbb{C}=\operatorname{Car}\left(\frac{\mathfrak{N}}{I \mathfrak{N}}\right) \times \mathbb{C}
$$

Part b) of the proposition follows from $\rho(\mathfrak{R})=\frac{\mathscr{D}_{r}}{\rho^{|g|}}$ where

$$
\rho(\mathscr{g})=\left\{P\left(y, \partial_{y}, \theta_{11}, \theta_{12 \ldots}, \theta_{1 q}, \ldots, \theta_{q 1}, \ldots, \theta_{q q}\right)_{\mid \theta_{y}=0}: P \in \mathscr{g}\right\} .
$$

Notation. For $k \in \mathbb{Z}$ the module $\mathscr{D}_{[\Lambda]}[k]$ is a coherent $\mathscr{D}_{[\Lambda]}[0]$-bimodule (in fact it is locally free). Therefore, given a coherent $\lambda_{*} \mathscr{D}_{[\Lambda]}$-module $\mathfrak{N}$, we may consider the coherent $\mathscr{D}_{Y}$-module

$$
\begin{aligned}
\mathfrak{N}_{Y, k} & =\mathscr{D}_{Y} \otimes_{\lambda_{*} \mathscr{D}_{111}[0]}\left(\lambda_{*} \mathscr{D}_{[\Lambda]}[k] \otimes_{\lambda_{*} \mathscr{D}_{11}[0]} \mathfrak{N}\right) \\
& =\rho\left(\lambda_{*} \mathscr{D}_{[\Lambda]}[k] \bigotimes_{\lambda_{*} \mathscr{I}_{11}[0]} \mathfrak{N}\right) .
\end{aligned}
$$


Example 3.3. Let $P \in F_{k}^{Y} \mathscr{D}_{X}$ and let $\mathfrak{N}=g r^{0}\left(\frac{\mathscr{D}_{X}}{\mathscr{D}_{X} P}\right)$, where $\frac{\mathscr{D}_{X}}{\mathscr{D}_{X} P}$ is equipped with the induced filtration $F^{Y} \mathscr{D}_{Y \rightarrow X}$. Then

$$
\begin{aligned}
\mathfrak{N}_{Y, k} & =\mathscr{D}_{Y} \otimes_{\lambda_{*} \mathscr{D}_{[1]}[0]}\left(\lambda_{*} \mathscr{D}_{[\Lambda]}[k] \otimes_{\lambda_{*} \mathscr{D}_{[11}[0]} g r^{0}\left(\frac{\mathscr{D}_{X}}{\mathscr{D}_{X} P}\right)\right) \\
& =\mathscr{D}_{Y} \otimes_{\lambda_{*} \mathscr{D}_{[1]}[0]} \frac{g r^{k}\left(\mathscr{D}_{X}\right)}{g r^{k}\left(\mathscr{D}_{X}\right) \sigma_{0}(P)} \\
& =\frac{g r^{k}\left(\mathscr{D}_{Y \rightarrow X}\right)}{g r^{k}\left(\mathscr{D}_{Y \rightarrow X}\right) \sigma_{0}(P)} .
\end{aligned}
$$

Example 3.4. Given $k \geq 1$ let $P \in F_{k}^{Y} \mathscr{D}_{X \mid Y} \backslash F_{k-1}^{Y} \mathscr{D}_{X \mid Y}$. Then, in the special local coordinate system chosen in the introductory section,

$$
P=Q+\sum_{|\beta|=k} \partial_{t}^{\beta} Q_{\beta}
$$

where $Q \in F_{k-1}^{Y} \mathscr{D}_{X}$ and $Q_{B} \in F_{0}^{Y} \mathscr{D}_{X}$. Thus, locally,

$$
g r^{0}\left(\frac{\mathscr{D}_{X}}{\mathscr{D}_{X} P}\right) \simeq \frac{g r^{0} \mathscr{D}_{X}}{\bigoplus_{|\alpha|=k} g r^{0}\left(\mathscr{D}_{X}\right) \tau^{\alpha} \sum_{|\beta|=k} \partial_{\tau}^{\beta} \widehat{\sigma}\left(Q_{B}\right)}
$$

Since

$$
\mathscr{D}_{Y}=\frac{g r^{0} \mathscr{D}_{X}}{g r^{0} \mathscr{D}_{X}\left(\tau_{1} \partial_{\tau 1}, \ldots, \tau_{1} \partial_{\tau q}, \ldots, \tau_{q} \partial_{\tau 1}, \ldots, \tau_{q} \partial_{\tau q}\right)},
$$

it follows that

$$
\begin{aligned}
\mathfrak{N}_{Y, 0} & =\mathscr{D}_{Y} \bigotimes_{g r \mathscr{D}_{\lambda}} g r^{0}\left(\frac{\mathscr{D}_{X}}{\mathscr{D}_{X} P}\right) \\
& =\frac{g r^{0} \mathscr{D}_{X}}{g r^{0} \mathscr{D}_{X}\left(\tau_{1} \partial_{\tau 1}, \ldots, \tau_{q} \partial_{\tau q}\right)} \otimes_{g r \mathscr{D}_{X}} g r^{0}\left(\frac{\mathscr{D}_{X}}{\mathscr{D}_{X} P}\right) \\
& =0 .
\end{aligned}
$$

Proposition 3.5. [LS87]

(i) Let $\mathfrak{M}$ be a coherent $\lambda_{*} \mathscr{D}_{[\Lambda]}$-module and let $\mathfrak{N}$ be a coherent sub$\lambda_{*} \mathscr{D}_{\Lambda]}[0]$-module of $\mathfrak{M}$ that generates $\mathfrak{M}$ over $\lambda_{*} \mathscr{D}_{[\Lambda]}$. Then

$$
\mathfrak{S}(\mathfrak{M}):=\underset{k \in \mathrm{Z}}{\cup} \operatorname{Car}\left(\mathfrak{N}_{Y, k}\right)
$$

is a subset of $T^{*} Y$ which does not depend on the choice of $\mathfrak{N}$.

(ii) If $\mathrm{O} \longrightarrow \mathfrak{M}^{\prime} \longrightarrow \mathfrak{M} \longrightarrow \mathfrak{M}^{\prime \prime} \longrightarrow 0$ is an exact sequence of coherent $\lambda_{*} \mathscr{D}_{[\Lambda]}$-modules then

$$
\mathfrak{S}(\mathfrak{M})=\mathfrak{S}\left(\mathfrak{M} \mathfrak{Z}^{\prime}\right) \cup \mathfrak{S}\left(\mathfrak{M}^{\prime \prime}\right) \text {. }
$$

Proof. (i ) Let $\mathfrak{N}$ and $\mathfrak{N}^{\prime}$ two coherent $\lambda * \mathscr{D}_{[\Lambda]}[0]$-modules that generate $\mathfrak{M}$. As $\mathfrak{M}$ is a generator of $\mathfrak{M}$ we have 


$$
\mathfrak{N}^{\prime}=\sum_{k \in \mathbf{Z}}\left(\lambda_{*} \mathscr{D}_{[\Lambda]}[k] \mathfrak{N}\right) \cap \mathfrak{N}^{\prime}
$$

and so $\mathfrak{N}^{\prime}=\underset{k \in \mathrm{N}}{\cup} \mathfrak{N}^{(k)}$ where

$$
\mathfrak{N}^{(k)}=\sum_{-k \leq \jmath \leq k}\left(\lambda_{*} \mathscr{D}_{[\Lambda]}[j] \mathfrak{N}\right) \cap \mathfrak{N}^{\prime}
$$

The sequence $\left(\mathfrak{N}^{\prime(k)}\right)_{k \in \mathbf{Z}}$ is a sequence of coherent $\lambda_{*} \mathscr{D}_{[\Lambda]}[0]$-modules of $\mathfrak{N}^{\prime}$, and being $\mathfrak{R}^{\prime}$ of finite type this sequence must stabilize. Let $k_{0}$ be an integer such that $\mathfrak{N}^{\prime}=\mathfrak{N}^{\left(k_{0}\right)}$ and let $\mathfrak{N}^{\prime \prime}=\sum_{-k_{0} \leq j \leq k_{0}}\left(\lambda_{*} \mathscr{D}_{[\Lambda]}[j] \mathfrak{R}\right)$.

Then

$$
\bigcup_{k \in \mathbf{Z}} \operatorname{Car}\left(\mathfrak{N}_{Y, k}^{\prime}\right) \subset \operatorname{Car}\left(\mathfrak{N}_{Y, k}^{\prime \prime}\right)=\underset{k \in \mathbf{Z}}{\cup} \operatorname{Car}\left(\mathfrak{N}_{Y, k}\right) .
$$

Reversing the roles of $\mathfrak{N}$ and $\mathfrak{N}^{\prime}$ we get the first part of the proposition.

(11) It is enough to prove that if $0 \longrightarrow \mathfrak{N} \longrightarrow \mathfrak{N} \longrightarrow \mathfrak{N}^{\prime \prime} \longrightarrow 0$ is an exact sequence of coherent $\lambda * \mathscr{D}_{[\Lambda]}[0]$-modules then

$$
\operatorname{Car}\left(\mathfrak{N}_{Y, k}\right)=\operatorname{Car}\left(\mathfrak{R}_{Y, k}^{\prime}\right) \cup \operatorname{Car}\left(\mathfrak{M}_{Y, k}^{\prime \prime}\right) .
$$

But this is an immediate consequence of Proposition 3.2 and of the flatness of $\lambda_{*} \mathscr{D}_{[\Lambda]}[k]$ over $\lambda_{*} \mathscr{D}_{i \Lambda 1}[0]$.

\section{Definition of $\operatorname{Car}_{Y}^{Z}(\mathfrak{M})$}

Now let $\Lambda=T_{Y} X \stackrel{\lambda}{\longrightarrow} Y$ be the normal bundle of $Y$ in $X$. Let $\mathfrak{M}$ be a coherent $\mathscr{D}_{X}$-module and let $F_{Y} \mathfrak{M}$ be a good filtration on $\mathfrak{M}$. Then the graded module for this filtration, $g r_{Y} \mathfrak{M}$, is a coherent $\mathscr{D}_{[\Lambda]}$-module and $\mathfrak{N}=g r_{Y}^{0}(\mathfrak{M})$ generates $\mathfrak{M}$ over $\mathscr{D}_{[\Lambda]}$. Thus we can associate to $g r_{Y}(\mathfrak{M})$ the subset $\mathfrak{S}\left(g r_{Y} \mathfrak{M}\right)$ of $T^{*} Y$. By Proposition (3.5), the functor $\mathfrak{N} \mapsto \mathfrak{S}(\mathfrak{N})$ is an additive one. By Proposition 1.3.1. of Chap. II of [Sch85], $\mathfrak{S}\left(g r_{Y} \mathfrak{M}\right)$ is independent of the choice of the good $F_{Y} \mathscr{D}_{X}$-filtration and the functor $\mathfrak{M} \mapsto \mathfrak{S}\left(g r_{Y} \mathfrak{M}\right)$ is an additive onc. Therefore we have the following proposition

Proposition 4.1. Let $\mathfrak{M}$ be a coherent $\mathscr{D}_{X}$ module and let $F_{Y} \mathfrak{M}$ be a good $F_{Y} \mathscr{D}_{X}$-filtration on $\mathfrak{M}$. Then

(i) $\mathfrak{S}\left(g r_{Y} \mathfrak{M}\right)$ is a subset of $T^{*} Y$ and does not depend on the choice of the good $F_{Y} \mathscr{D}_{X}$-filtration on $\mathfrak{M}$.

(ii) if $0 \longrightarrow \mathfrak{M}^{\prime} \longrightarrow \mathfrak{M} \longrightarrow \mathfrak{M}^{\prime \prime} \longrightarrow 0$ is an exact sequence coherent $\mathscr{D}_{X}$-modules then

$$
\mathfrak{S}\left(g r_{Y} \mathfrak{M}\right)=\mathfrak{S}\left(g r_{Y} \mathfrak{M}^{\prime}\right) \cup \mathfrak{S}\left(g r_{Y} \mathfrak{M}^{\prime \prime}\right)
$$

This proposition enables us to make the following definition, as in [LS87]: 
Definition 4.2. Let $\mathfrak{M}$ be a coherent $\mathscr{D}_{X}$-module and let $F_{Y} \mathfrak{M}$ be a good $F_{Y} \mathscr{D}_{X}$-filtration on $\mathfrak{M}$. We define

$$
\operatorname{Car}_{Y}^{\mathbb{Z}}(\mathfrak{M}):=\mathfrak{S}\left(\operatorname{gr}_{Y} \mathfrak{M}\right)=\underset{k \in \mathbb{Z}}{\cup} \operatorname{Car}\left(\mathfrak{N}_{Y, k}\right)
$$

where $\mathfrak{N}_{Y, k}=\mathscr{D}_{Y} \bigotimes_{\lambda_{*} \mathscr{D}_{[\Lambda 1}[0]} \lambda_{*} \mathscr{D}_{[\Lambda]}[k] \otimes_{\lambda_{*} \mathscr{D}_{[\Lambda]}[0]} g r_{Y}^{0}(\mathfrak{M})$

The goal of the remaining sections is to prove that $\operatorname{Car}_{Y}^{\mathbf{Z}}(\mathfrak{M})$ is an upper bound for $\operatorname{Car}\left(\mathfrak{M}_{Y}^{\circ}\right)$.

\section{The Case Where $\mathfrak{M}$ is the Hodule Defined by Ome Operator}

Let $P \in \mathscr{D}_{X \mid Y}$ and $\mathfrak{M}=\mathscr{D}_{X} / \mathscr{D}_{X} P$. Then

$$
0 \longrightarrow \mathscr{D}_{X} \stackrel{P}{\longrightarrow} \mathscr{D}_{X} \longrightarrow \mathfrak{M} \longrightarrow 0
$$

is a free resolution of $\mathfrak{M}$. So, in the derived category,

$$
\mathfrak{M}_{Y}^{\circ} \simeq \mathscr{D}_{Y \rightarrow X} \stackrel{P}{\longrightarrow} \mathscr{D}_{Y \rightarrow X} \simeq \frac{\mathscr{D}_{X}}{\mathscr{J}} \stackrel{P}{\longrightarrow} \frac{\mathscr{D}_{X}}{\mathscr{J}}
$$

and

$$
\mathfrak{M}_{Y}^{0}=\mathbb{K} \mathbb{e r} P, \quad \mathfrak{M}_{Y}^{1}=\mathbb{C o k \mathbb { e }} P .
$$

Let $P \in F_{Y}^{0} \mathscr{D}_{X}$. Then $\left(F_{Y}^{k} \mathscr{D}_{Y \rightarrow X}\right) . P \subset F_{Y}^{k} \mathscr{D}_{Y \rightarrow X}$. Hence we can define

$$
F_{Y}^{k} \mathscr{D}_{Y \rightarrow X} \stackrel{\hat{\rho}_{k}(P)}{\longrightarrow} F_{Y}^{k} \mathscr{D}_{Y \rightarrow X}
$$

where $\widehat{\rho}_{k}(P)$ is the restriction of $P$ to $F_{Y}^{k} \mathscr{D}_{Y \rightarrow X}$.

Consider now the action of $P$ on the vectors $\delta^{\alpha}$ of the base $\left(\delta^{\gamma}\right)_{|r| \leq k}$ of $F_{Y}^{k} \mathscr{D}_{Y \rightarrow X}=\bigoplus_{|\gamma| \leq k} \mathscr{D}_{Y} \delta^{\gamma}$. If $P$ is locally formally written as

$$
P=\sum_{|\alpha| \geq|\beta|} P_{\alpha, \beta}\left(y, \partial_{y}\right) t^{\alpha} \partial_{t}^{\beta}
$$

then

$$
\delta^{\gamma} . P=\sum_{|\alpha| \geq|\beta|} P_{\alpha, \beta}\left(y, \partial_{y}\right) \frac{\gamma !}{(\gamma-\alpha) !} \delta^{\gamma-\alpha+\beta}
$$

Let $A(\gamma, \theta)$ the coefficient of $\delta^{\theta}$ in the expression of $\delta^{\gamma} . P$. Then

$$
A(\gamma, \theta)=\sum_{0 \leq \alpha \leq r} P_{\alpha, \theta-\gamma+\alpha}\left(y, \partial_{y}\right) \frac{\gamma !}{(\gamma-\alpha) !} \text {. }
$$

Ordering the base $\left(\delta^{r}\right)_{|r| \leq k}$ in such a way that all the $\delta^{\gamma}$ with $|\gamma|=i$ have orders lower than the $\delta^{r}$ with $|\gamma|=i+1$, it follows that the matrix $A(k)$ of $\hat{\rho}(k)$ in such a base is block-lower-triangular: 


$$
A(k)=\left(\begin{array}{cccc}
A_{00} & 0 & \cdots & 0 \\
A_{10} & A_{11} & \cdots & 0 \\
\cdots \cdots \cdots \cdots \cdots \cdots \cdots & \cdots \cdots \cdots \\
A_{k 0} & A_{k 1} & \cdots & A_{k k}
\end{array}\right)
$$

where $A_{\imath}$ is the matrix $A(k)(\gamma, \theta)_{|\gamma|=\imath,|\theta|=\jmath}$. Clearly that $A(k-1)$ is the matrix $A(k)$ with the last row and last column of blocks omitted. Thus

$$
\operatorname{Car}\left(\frac{F_{Y}^{k} \mathscr{D}_{Y \rightarrow X}}{F_{Y}^{k} \mathscr{D}_{Y \rightarrow X} \widehat{\rho}_{k}(P)}\right)=\bigcup_{j=1}^{k}\left\{y^{*} \in T^{*} Y: \quad \operatorname{det}\left(A_{j j}\right)\left(y^{*}\right)=0\right\}
$$

where det is the determinant of Sato-Kashiwara [SK75].

But $\left\{y^{*} \in T^{*} Y: \operatorname{det}\left(A_{k k}\right)\left(y^{*}\right)=0\right\}$ is precisely $\operatorname{Car}\left(\frac{g_{Y}^{*}\left(\mathscr{D}_{Y-1}\right)}{g^{*} \mathscr{Y}_{Y}\left(\mathscr{D}_{Y-1}\right) \rho_{k}(P)}\right)$ where $g r_{Y}^{k}\left(\mathscr{D}_{Y \rightarrow X}\right) \stackrel{\rho_{k}(P)}{\longrightarrow} g r_{Y}^{k}\left(\mathscr{D}_{Y \rightarrow X}\right)$ is the linear morphism whose matrix in base $\left(\delta^{r}\right)_{|r|=k}$ is $A_{k k}$. Observe now that $\rho_{k}(P)$ is the morphism $g r_{Y}^{k} \mathscr{D}_{Y \rightarrow X} \stackrel{\sigma_{0}(P)}{\longrightarrow} g \gamma_{Y}^{k} \mathscr{D}_{Y \rightarrow X}$. Then, from example (3.4) it follows that

$$
\text { - } P \in F_{Y}^{0} \mathscr{D}_{X \mid Y} \Rightarrow \operatorname{Car}\left(\left(\frac{\mathscr{D}_{X}}{\mathscr{D}_{X} \cdot P}\right)_{Y}^{\mathscr{D}}\right)=\bigcup_{k \in \mathrm{N}} \rho\left(\lambda_{*} \mathscr{D}_{[\Lambda]}[k] \otimes_{\lambda_{+} \mathscr{D}_{[\Lambda}[0]} g r_{Y}^{0} \frac{\mathscr{D}_{X}}{\mathscr{D}_{X} . P}\right)
$$

and thus the following property is true:

$$
\text { - } P \in F_{Y}^{0} \mathscr{D}_{X \mid Y} \Rightarrow\left\{\begin{array}{l}
\operatorname{Car}\left(\mathfrak{M}_{Y}^{\ominus}\right) \subset \operatorname{Car}_{Y}^{\mathbf{Z}}(\mathfrak{M}) \\
\operatorname{Car}_{Y}^{Z}(\mathfrak{M})=\underset{k \in \mathbf{Z}}{\bigcup}\left\{y^{*} \in T^{*} Y: \quad \operatorname{det}\left(\rho_{k}(P)\right)\left(y^{*}\right)=0\right\}
\end{array}\right.
$$

From example (3.3) and from definition of $\operatorname{Car}_{Y}^{\mathbf{Z}}(\mathfrak{M})$ it follows that

- $k>0$ and $P \in F_{Y}^{k} \backslash F_{Y}^{k-1} \mathscr{D}_{X} \Rightarrow \operatorname{Car}_{Y}^{\mathbb{Z}}(\mathfrak{M})=T^{*} Y$.

Hence we have proved the following proposition

Proposition 5.1. Let $P \in \mathscr{D}_{X \mid Y}$ and $\mathfrak{M}=\frac{\mathscr{D}_{1}}{\mathscr{D}_{1} P} . \quad$ Then

(a) $\operatorname{Car}\left(\mathfrak{M}_{Y}^{\bullet}\right) \subset \operatorname{Car} \underset{Y}{\mathrm{Z}}(\mathfrak{M})$.

(b) Moreover if $P \in F_{Y}^{0} \mathscr{D}_{X}$ then

$$
\operatorname{Car}_{Y}^{Z}(\mathfrak{M})=\bigcup \underset{k \in \mathbf{Z}}{\cup}\left\{y^{*} \in T^{*} Y: \quad \operatorname{det}\left(\rho_{k}(P)\right)\left(y^{*}\right)=0\right\} .
$$

\section{The Case Where $\mathfrak{M}$ is the Module Defined by a Coherent Ideal}

Let $\mathscr{J}$ be a coherent ideal of $\mathscr{D}_{X}$; then $g r_{Y}\left(\frac{\mathscr{D}_{Y}}{\mathscr{I}}\right)$ is generated by $g r_{Y}^{0}\left(\frac{F_{Y}^{\mathscr{D}_{1}}}{\mathscr{D}^{0}}\right)$ where $\mathscr{g}_{0}=\mathscr{g} \cap F_{Y}^{0} \mathscr{D}_{X}$. Therefore

$$
\operatorname{Car}_{Y}^{\mathbf{Z}}\left(\frac{\mathscr{D}_{X}}{\mathscr{J}}\right)=\underset{k \in \mathbf{Z}}{\cup} \operatorname{Car}\left(\left(\mathscr{D}_{Y} \otimes_{\lambda_{*} \mathscr{D}_{[\Lambda]}[0]} \lambda_{*} \mathscr{D}_{[\Lambda]}[k]\right) \bigotimes_{\lambda_{*} \mathscr{D}_{[1]}[0]} g r_{Y}^{0}\left(\frac{F_{Y}^{0} \mathscr{D}_{X}}{\mathscr{J}_{0}}\right)\right)
$$




$$
\begin{aligned}
& =\bigcup_{k \in \mathbb{Z}} \operatorname{Car}\left(g r^{k} \mathscr{D}_{Y \rightarrow X} \otimes_{\lambda_{*} \mathscr{D}_{[\Lambda]}[0]} \frac{\lambda_{*} \mathscr{D}_{[A]}[0]}{g r_{Y}^{0}\left(\mathscr{g}_{0}\right)}\right) \\
& =\bigcup_{k \in \mathbb{Z}} \operatorname{Car}\left(\frac{g r^{k} \mathscr{D}_{Y \rightarrow X}}{g r_{Y}^{0}\left(\mathscr{g}_{0}\right)}\right)
\end{aligned}
$$

Let $P$ be an element of $\mathscr{g}$ and let $\sigma_{0}(P)$ be the image of $P$ in $g r_{Y}^{0}\left(\mathscr{g}_{0}\right)$. Then

$$
\frac{g r^{k} \mathscr{D}_{Y \rightarrow X}}{g r^{k} \mathscr{D}_{Y \rightarrow X} \sigma_{0}(P)} \longrightarrow \frac{g r^{k} \mathscr{D}_{Y \rightarrow X}}{g r^{k} \mathscr{D}_{Y \rightarrow X} g r_{Y}^{0}\left(\Phi_{0}\right)} \longrightarrow 0,
$$

is an exact sequence of $g r^{0} \mathscr{D}_{Y \rightarrow X}$-modules. Thus, taking into account equation (3),

$$
\operatorname{Car}_{Y}^{\mathrm{Z}}\left(\frac{\mathscr{D}_{X}}{\mathscr{g}}\right) \subset \underset{P \in \mathscr{g}}{\cap} \operatorname{Car}_{Y}^{\mathrm{Z}}\left(\frac{\mathscr{D}_{X}}{\mathscr{D}_{X} \cdot P}\right)
$$

i.e.

$$
\operatorname{Car}_{Y}^{\mathbb{Z}}\left(\frac{\mathscr{D}_{X}}{\mathscr{J}}\right) \subset \underset{k \in \mathbb{Z}}{\cup}\left\{y^{*} \in T^{*} Y: \operatorname{det}\left(\rho_{k}(P)\right)\left(y^{*}\right)=0 \quad \forall P \in \mathscr{G}\right\} .
$$

The following proposition shows that the above inclusion is in fact an equality.

Proposition 6.1. Let $\mathscr{J}$ be a coherent ideal of $\mathscr{D}_{X}$. Then

$$
\operatorname{Car}_{Y}^{Z}\left(\frac{\mathscr{D}_{X}}{\mathscr{g}}\right)=\bigcup_{k \in \mathbf{Z}}\left\{y^{*} \in T^{*} Y: \operatorname{det}\left(\rho_{k}(P)\right)\left(y^{*}\right)=0 \quad \forall P \in \mathscr{g}\right\} .
$$

Proof. For each $k \in \mathbb{Z}$ let us denote by $\mathscr{L}_{k}$ the following $\mathscr{D}_{Y}$-module:

$$
\mathscr{L}_{k}=\mathscr{D}_{Y} \otimes_{\lambda_{+} \mathscr{D}_{M 1}[0]} \lambda_{*} \mathscr{D}_{[\Lambda]}[k]=\left\{\begin{array}{cl}
\bigoplus_{|\alpha|=k} \mathscr{D}_{Y} \partial_{t}^{\alpha} & \text { if } k \geq 0 \\
\bigoplus_{|\alpha|=-k} \mathscr{D}_{Y} t^{\alpha} & \text { if } k \leq 0
\end{array} .\right.
$$

Then we have a commutative diagram of ring homomorphisms

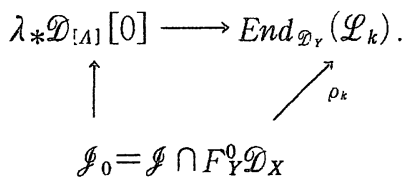

We denote by $\mathcal{N}_{k}$ the $\mathscr{D}_{Y}$-module $\mathscr{L}_{k} \rho_{k}\left(\mathscr{J}_{0}\right)$. With this notation, we see that, to prove the proposition, it is enough to prove that, for all $k \in \mathbb{Z}$,

$$
\operatorname{Car}\left(\frac{\mathscr{L}_{k}}{\mathcal{N}_{k}}\right) \supset \bigcap_{Q \in \rho_{k}\left(\mathscr{L}_{0}\right)} \operatorname{Car}\left(\frac{\mathscr{L}_{k}}{\mathscr{L}_{k} Q}\right)
$$

Let $e_{1}, \ldots, e_{s}$ be a basis of the free $\mathscr{D}_{Y}$-module $\mathscr{L}_{k}$ and we write $\mathscr{L}_{k}=\bigoplus_{i=1}^{s} \mathscr{D}_{Y} e_{\imath}$. We denote by $\mathscr{A}_{k}$ the ring of $\mathscr{D}_{Y}$-endomorphisms $\operatorname{End}_{D_{Y}}\left(\mathscr{L}_{k}\right)$. Then $\mathscr{L}_{k}$ is a 
left-right $\left(\mathscr{D}_{Y}, \mathscr{A}_{k}\right)$-bimodule and the functor

$$
\begin{aligned}
\operatorname{Mod}\left(\mathscr{D}_{Y}\right) & \stackrel{\operatorname{Hom}\left(\mathscr{L}_{k}\right)}{\longrightarrow} \operatorname{Mod}\left(\mathscr{A}_{k}\right) \\
\mathfrak{M} & \longmapsto \operatorname{Hom}_{\mathscr{D}_{Y}}\left(\mathscr{L}_{k}, \mathfrak{M}\right)
\end{aligned}
$$

is an equivalence of categories. The correspondence

$$
\begin{aligned}
\operatorname{Mod}\left(\mathscr{A}_{k}\right) & \stackrel{\mathscr{L}_{k} \bigotimes_{\mathscr{A}_{k}}}{\longrightarrow} \operatorname{M} \text { od }\left(\mathscr{D}_{Y}\right) \\
\mathscr{Q} & \longmapsto \mathscr{L}_{k} \bigotimes_{\mathscr{A}_{k}} \mathscr{Q}
\end{aligned}
$$

is a left adjoint functor. This gives a correspondence

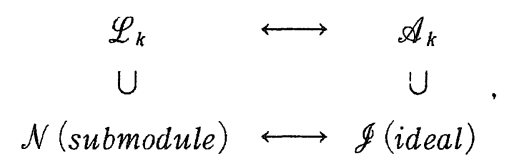

between submodules of $\mathscr{L}_{k}$ and ideals of $\mathscr{A}_{k}$. Thus one has a bijective correspondence

$$
\frac{\mathscr{L}_{k}}{\mathscr{L}_{k} \mathscr{J}}=\mathscr{L}_{k} \otimes_{\mathscr{A}_{k}}\left(\frac{\mathscr{A}_{k}}{\mathscr{J}}\right) \longleftrightarrow \frac{\mathscr{A}_{k}}{\mathscr{J}}
$$

For each $\left(u_{1}, \ldots, u_{s}\right) \in \mathscr{L}_{k}^{\oplus_{s}}$ let $\phi\left(u_{1}, \ldots, u_{s}\right): \mathscr{L}_{k} \longrightarrow \mathscr{L}_{k}$ be the homomorphism of free $\mathscr{D}_{Y}$-modules defined by $\phi\left(u_{1}, \ldots, u_{s}\right)\left(e_{\imath}\right)=u_{i}$. Then $\mathscr{L}_{k}^{\oplus s} \longrightarrow \mathscr{A}_{k},\left(u_{1}, \ldots, u_{s}\right)$ $\mapsto \phi\left(u_{1}, \ldots, u_{s}\right)$ is an isomorphism. Now $\mathscr{g} \simeq \mathcal{N}_{k}^{\oplus s}$. Hence we have

$$
\bigcap_{P \in \rho_{k}\left(\mathscr{g}_{0}\right)} \operatorname{Car}\left(\frac{\mathscr{L}_{k}}{\mathscr{L}_{k} . P}\right)=\bigcap_{\left(u_{1}, \ldots, u_{s}\right) \in N_{k}}^{\oplus_{s}} \operatorname{Car}\left(\frac{\mathscr{L}_{k}}{\sum_{i=1}^{s} \mathscr{D}_{Y} u_{\imath}}\right)
$$

Suppose now that $p \in T^{*} Y \backslash \operatorname{Car}\left(\frac{\mathscr{Q}_{k}}{\mathcal{N}_{k}}\right)$. Then there is some $Q \in \mathscr{D}_{Y}$ such that $Q e_{2} \in$ $\mathcal{N}_{k}(1 \leq i \leq s)$ and $\sigma(Q)(p) \neq 0$. Setting $u_{\imath}=Q e_{\imath}(i=1, \ldots s)$ it follows that $\frac{\mathscr{L}_{k}}{\sum_{i=1}^{s} \mathscr{D}_{\gamma u_{t}}}$ is isomorphic as a $\mathscr{D}_{Y}$-module to $\underset{i=1}{\bigoplus_{i=1}^{S}} \frac{\mathscr{D}_{Y}}{\mathscr{D}_{Y} Q}$, implying $p \notin \operatorname{Car}\left(\frac{\mathscr{L}_{k}}{\sum_{i=1}^{S} \mathscr{D}_{Y t_{i}}}\right)$.

\section{Main Theorem}

Now everything is prepared for the statement and proof of the main theorem.

Theorem 7.1. Let $X$ be a complex analytic manifold, $Y$ a smooth submanifold of $X$ and $\mathfrak{M}$ a coherent $\mathscr{D}_{X}$-module. Then

$$
\operatorname{Car}\left(\mathfrak{M}_{Y}^{\bullet}\right) \subset \operatorname{Car}_{Y}^{Z}(\mathfrak{M})
$$


Proof. Let $\theta$ be a point in $T^{*} Y \backslash \operatorname{Car}_{Y}^{\mathbb{Z}}(\mathfrak{M})$. Given a section $u$ of $\mathfrak{M}$ let $\mathscr{g} \subset$ $\mathscr{D}_{X}$ be the annihilator of $u$. By proposition (6.1) there exists a $P \in \mathscr{J}$ such that $\operatorname{det}\left(\rho_{k}(P)\right)(\theta) \neq 0$ for all $k \in \mathbb{Z}$. In fact, if this was not the case, then we would have $\theta \in \operatorname{Car}_{Y}^{Z}\left(\frac{\mathscr{D}_{X}}{\mathscr{g}}\right)$ and, since $\mathscr{D}_{X} / \mathscr{J} \longrightarrow \mathfrak{M}, P \mapsto P . u$ is an injective morphism, one would conclude by proposition (4.1) that $\theta \in \operatorname{Car}_{Y}^{Z}(\mathfrak{M})$.

As the module $\mathfrak{M}$ is locally of finite type there is a local system of generators $\left(u_{1}, \ldots, u_{s}\right)$ of $\mathfrak{M}$ and for each $u_{\imath}$ one operator $P_{\imath}$, such that $P_{\imath} u_{\imath}=0$ and $\operatorname{det}\left(\rho_{k}\left(P_{\imath}\right)\right)(\theta) \neq 0$ for all $k \in \mathbb{Z}$ and $1 \leq i \leq s$.

Let us denote $\mathscr{L}=\bigoplus_{i=1}^{S} \frac{\mathscr{D}_{X}}{\mathscr{D}_{X} P_{t}}$ and let $\mathscr{L} \stackrel{\phi}{\longrightarrow} \mathfrak{M}$ be the morphism that sends $u_{\imath}$ to the class of 1 modulo $\mathscr{D}_{X} . P_{\imath}$. Let $\mathcal{N}$ be $\mathbb{K} e r(\phi)$. Then there is an exact sequence of left $\mathscr{D}_{X}$-modules:

$$
0 \longrightarrow \mathcal{N} \longrightarrow \mathscr{L} \longrightarrow \mathfrak{M} \longrightarrow 0 \text {. }
$$

Applying the functor $\mathscr{D}_{Y \rightarrow X} \otimes_{\mathscr{D}_{\lambda}}$ to the above exact sequence we get a long exact sequence of cohomology

$$
\cdots \rightarrow \mathcal{N}_{Y}^{k} \longrightarrow \mathscr{L}_{Y}^{k} \longrightarrow \mathfrak{M}_{Y}^{k} \longrightarrow \cdots \longrightarrow \mathfrak{M}_{Y}^{0} \longrightarrow 0 .
$$

As the theorem was already proved for modules of type $\frac{\mathscr{D}_{X}}{\mathscr{D}_{\lambda} P}$ and there exists a $k_{0} \in \mathbb{Z}$ such that $\mathcal{N}^{k_{0}}=0$, one may assume, as an induction hypothesis, that $\operatorname{Car}\left(\mathfrak{M}_{Y}^{k}\right) \subset \operatorname{Car} \mathbb{Z}(\mathfrak{M})$ for all coherent $\mathscr{D}_{X}$-module $\mathfrak{M}$ and all $k \leq k_{0}$. Now, from the long exact sequence (6), it follows that

$$
\operatorname{Car}\left(\mathfrak{M}_{Y}^{k_{0}+1}\right) \subset \operatorname{Car}\left(\mathscr{L}_{Y}^{k_{0}+1}\right) \cup \operatorname{Car}\left(\mathcal{N}_{Y}^{k_{0}}\right),
$$

implying that $\theta \notin \operatorname{Car}\left(\mathbb{M}_{Y}^{k_{0}+1}\right)$. Hence, by induction, we finally conclude that

$$
\theta \notin \underset{k \in \mathbb{N}}{\cup} \operatorname{Car}\left(\mathfrak{M}_{Y}^{-k}\right)=: \operatorname{Car}\left(\mathfrak{M}_{Y}^{\circ}\right),
$$

finishing the proof of the theorem.

\section{References}

[Ka83a] Kashiwara. M.. Systems of Microdifferential equations. Notes by T. Monteiro Fernandes. Progress in Math. 34, Birkhauser (1983)

[Ka83b] Vanishing cycles and holonomic systems of differential equations, Lecture Notes in Math., Sprınger Verlag, 1016 (1983), 134-142.

[SK75] Sato. M. and Kashiwara. M., The determinant of the matrices of the pseudo-differential operators, Proc. Japan Acad., $5 \mathbb{1}$ (1975).

[Sch85] Schapira, P., Microdıfferential Systems in the complex domain, Grundlehren Math. Wiss., Springer Verlag, 269 (1985)

[LS87] Laurent, Y. and Schapıra, P., Images Inverses des Modules differentiels. Compositıo Math., 61 (1987), 229-251. 